UNIVERSITÀ DEGLI STUDI DI BERGAMO

DIPARTIMENTO DI INGEGNERIA DELL'INFORMAZIONE

E METODI MATEMATICI

QUADERNI DEL DIPARTIMENTO

Department of Information Technology and Mathematical Methods

Working Paper

Series "Mathematics and Statistics"

n. $7 / \mathrm{MS}-2012$

Prescription of general defective boundary

conditions in fluid-dynamics

by

L. Formaggia, C. Vergara 
COMITATO DI REDAZIONE ${ }^{\S}$

Series Information Technology (IT): Stefano Paraboschi

Series Mathematics and Statistics (MS): Luca Brandolini, Ilia Negri

\footnotetext{
$\S^{\S}$ 'accesso alle Series è approvato dal Comitato di Redazione. I Working Papers della Collana dei Quaderni del Dipartimento di Ingegneria dell'Informazione e Metodi Matematici costituiscono un servizio atto a fornire la tempestiva divulgazione dei risultati dell'attività di ricerca, siano essi in forma provvisoria o definitiva.
} 


\title{
Prescription of general defective boundary conditions in fluid-dynamics
}

\author{
Luca Formaggia and Christian Vergara
}

\begin{abstract}
This work reviews and extends to a more general setting some strategies to impose defective boundary conditions to fluid-dynamic problems investigated by the authors in the last years. We focus here to the steady Stokes problem as a paradigm for the unsteady and nonlinear cases. We show the well posedness of the proposed approaches and discuss their relative benefits.
\end{abstract}

Mathematics Subject Classification (2010). Primary 65N30; Secondary $76 \mathrm{D} 07$.

Keywords. Defective boundary conditions, flow rate, mean pressure, incompressible fluids, finite elements.

\section{Introduction and motivation}

A major attention has been paid lately to computational tools to give answers to medical doctors and bio-engineers on the behavior of the cardiovascular environment (see, e.g., $[10,12,13])$. These tools usually need to compute the blood flow in major arteries. To obtain significant patient-specific results, two are the crucial points: i) the reconstruction of the real geometry of the artery at hand, to build a patient-specific computational domain, and ii) the prescription of suitable boundary conditions at the artificial sections that are necessarily introduced when selecting the domain of interest.

This work is motivated by the second aspect, namely the problem of the prescription of appropriate boundary conditions. Indeed, in this type of applications usually one has at disposal only "defective" data, typically flow rates or mean pressure, coming either from measurements or from the coupling with reduced models (see, for instance [12]). This leads to the need

This work has been (partially) supported by the ERC Advanced Grant N.227058 MATHCARD and by the Italian MIUR PRIN09 project n. 2009Y4RC3B_001. 
of prescribing on one or more sections $\Sigma \subset \partial \Omega$ a condition of the type

$$
\alpha \rho_{f} \int_{\Sigma} \mathbf{u} \cdot \mathbf{n} d \sigma+\frac{1-\alpha}{|\Sigma|} \int_{\Sigma}(-p+\delta \mu(\nabla \mathbf{u} \mathbf{n}) \cdot \mathbf{n}) d \sigma=M,
$$

where $\alpha \in[0,1]$ and $M$ are given data and $\rho_{f}$ is the fluid density, whilst $\delta$ can assume the values 0 or 1 . The first integral on (1.1) is the flow rate. As for the second term, for $\delta=0$ we have the mean pressure, whilst for $\delta=1$ the mean normal component of the normal stress (in the following we indicate it simply by mean normal stress). The parameter $\alpha$ has been introduced to consider a general defective boundary condition, which includes two classical defective conditions, namely the flow rate $(\alpha=1)$ and the mean pressure or normal stress condition $(\alpha=0)$. These cases have been tackled in the context of Navier-Stokes equations in [17, 7, 3, 16] (even with fluid-structure interaction, see [5]) and also applied to practical haemodynamic problems, like in [19].

Here, we consider the general condition (1.1) for steady Stokes flow, since the main characteristics of the problem may already be put into evidence in this simplified setting. For the sake of simplicity, we also consider (1.1) applied only on a single section of the boundary, the extension to multiple sections being immediate.

Remark 1.1. As observed in $[17,21]$ for the case of the flow rate, a vectorial defective boundary condition could be also considered. For the general case, this is obtained by considering, together with condition (1.1), also the tangential conditions

$$
\alpha_{j} \rho_{f} \int_{\Sigma} \mathbf{u} \cdot \tau_{j} d \sigma+\frac{1-\alpha_{j}}{|\Sigma|} \int_{\Sigma} \delta \mu(\nabla \mathbf{u} \mathbf{n}) \cdot \tau_{j} d \sigma=M_{j}, \quad j=1,2,
$$

for suitable $\alpha_{j}$ and $M_{j}$. This could be the case, for example, of a coupling with a reduced 1D model where also information about the tangential velocity is included. However, for the sake of exposition, in what follows we focus just on condition (1.1).

The meaning of an $0<\alpha<1$ is manifold. For instance in the case of coupling with reduced models of the cardiovascular system, like the classical Windkessel model, $\alpha$ may be related to the resistance $R$ of the peripheral flow, by the relation $\alpha=\frac{R}{R+1}$. Another interpretation of $\alpha$ could be related to the reliability of flow rate and pressure measurements obtained on the same section $\Sigma$. If we have both measurement at disposal we can take this into account by weighting them in a different manner.

In this work, we review and compare four possible treatment of condition (1.1). In particular, in Section 2, we introduce the problem, whilst in Section 3 , we present four different strategies for its numerical treatment. Section 4 gives a critical comparison of the various methodologies. 


\section{Preliminaries}

Let $\Omega \subset \mathbb{R}^{d}, d=2,3$, be an open bounded domain with Lipschitz boundary, and let $\Sigma \subset \partial \Omega$ a measurable and connected portion of its boundary. We consider the following steady-Stokes problem:

Given the scalars $\alpha \in[0,1]$ and $M$ and the function $\mathbf{f} \in \mathbf{L}^{2}(\Omega)$, find $\mathbf{u} \in$ $\mathbf{V} \subset \mathbf{H}^{1}(\Omega)$ and $p \in Q=L^{2}(\Omega)$, such that

$$
\begin{cases}\mu \triangle \mathbf{u}+\nabla p=\mathbf{f} & \text { a.e. in } \Omega \\ \nabla \cdot \mathbf{u}=0 & \text { a.e. in } \Omega \\ \mathbf{u}=\mathbf{0} & \text { on } \Gamma_{D}, \\ \mu \nabla \mathbf{u} \mathbf{n}=\mathbf{0} & \text { on } \Gamma_{N} \\ \alpha \rho_{f} \int_{\Sigma} \mathbf{u} \cdot \mathbf{n} d \sigma+\frac{1-\alpha}{|\Sigma|} \int_{\Sigma}(-p+\delta \mu(\nabla \mathbf{u} \mathbf{n}) \cdot \mathbf{n}) d \sigma=M, & \end{cases}
$$

where $\mu>0$ is the constant viscosity and $\Gamma_{N}$ and $\Gamma_{D}$ two non overlapping portions of boundary, such that $\Gamma_{N} \cup \Gamma_{D} \cup \Sigma \equiv \partial \Omega$. The Neumann portion of the boundary is required to give meaning to the problem since the divergence free constraints leads to

$$
\int_{\partial \Omega} \mathbf{u} \cdot \mathbf{n} d \sigma=0
$$

and the absence of a Neumann boundary would reduce (1.1) to a mean stress condition for any value of $\alpha>0$ and would lead to a non solvable problem for $\alpha=1$ and $M \neq 0$. For this reason we require here that $\left|\Gamma_{N}\right|>0$. This condition is not necessary anymore if we consider more than one artificial section where a defective condition of the type (1.1) is imposed.

Of course, problem (2.1) has not a unique solution. Among all possible solutions, we focus on those obtained by making the following hypothesis.

Assumption 1. The normal stress on $\Sigma$ is aligned with the normal direction and it is constant over the section, that is

$$
-p \mathbf{n}+\mu \nabla \mathbf{u} \mathbf{n}=c \mathbf{n} \quad \text { on } \Sigma,
$$

for a suitable $c=c(\alpha) \in \mathbb{R}$.

We have the following result

Lemma 2.1. Under Assumption 1, there exists a unique scalar $c$ such that problem (2.1) admits a unique solution, for $\delta=1$.

Proof. In [7] and [15] the authors have demonstrated the well posedness for the mean stress and the flow rate condition problems, respectively (both satisfying Assumption 1). We consider then just the case $0<\alpha<1$. We can construct the two problems

$$
\begin{cases}-\mu \triangle \mathbf{u}_{0}+\nabla p_{0}=\mathbf{f} & \text { a.e. in } \Omega \\ \nabla \cdot \mathbf{u}_{0}=0 & \text { a.e. in } \Omega \\ \mathbf{u}_{0}=\mathbf{0} & \text { on } \Gamma_{D} \\ \mu \nabla \mathbf{u}_{0} \mathbf{n}=\mathbf{0} & \text { on } \Sigma \cup \Gamma_{N}\end{cases}
$$


and

$$
\begin{cases}-\mu \triangle \mathbf{u}_{1}+\nabla p_{1}=\mathbf{0} & \text { a.e. in } \Omega \\ \nabla \cdot \mathbf{u}_{1}=0 & \text { a.e. in } \Omega, \\ \mathbf{u}_{1}=\mathbf{0} & \text { on } \Gamma_{D} \\ \mu \nabla \mathbf{u}_{1} \mathbf{n}=\mathbf{0} & \text { on } \Gamma_{N} \\ -p_{1} \mathbf{n}+\delta \mu\left(\nabla \mathbf{u}_{1} \mathbf{n}\right)=\mathbf{n} & \text { on } \Sigma .\end{cases}
$$

The solution $(\mathbf{u}, p)$ of $(1.1)$ may be build by $(\mathbf{u}, p)=\left(\mathbf{u}_{0}, p_{0}\right)+\gamma\left(\mathbf{u}_{1}, p_{1}\right)$, where $\gamma=\frac{M+(\alpha-1) s_{0}-\alpha q_{0}}{\alpha\left(q_{1}-1\right)+1}$, having set, for $i=0,1$,

$$
q_{i}=\int_{\Sigma} \mathbf{u}_{i} \cdot \mathbf{n} d \sigma, \quad \text { and } s_{i}=|\Sigma|^{-1} \int_{\Sigma}\left(-p_{i}+\delta \mu\left(\nabla \mathbf{u}_{i} \mathbf{n}\right) \cdot \mathbf{n}\right) d \sigma .
$$

Indeed, we may verify easily that $(\mathbf{u}, p)$ satisfies the given problem. We only need to ensure that $\alpha\left(q_{1}-1\right)+1 \neq 0$. To do this, we write the weak formulation of (2.4) and we take $\mathbf{u}_{1}$ as test function. We obtain

$$
\mu \int_{\Omega} \nabla \mathbf{u}_{1}^{2} d \mathbf{x}-\int_{\Sigma}\left(-p_{1} \mathbf{n}+\delta \mu\left(\nabla \mathbf{u}_{1} \mathbf{n}\right)\right) \cdot \mathbf{u}_{1} d \sigma=\mu\left\|\nabla \mathbf{u}_{1}\right\|^{2}-\int_{\Sigma} \mathbf{u}_{1} \cdot \mathbf{n} d \sigma
$$

which leads to $q_{1}=\mu\left\|\nabla \mathbf{u}_{1}\right\|^{2}>0$. This, together with $0<\alpha<1$, implies $\alpha\left(q_{1}-1\right)+1 \neq 0$.

We observe also that the solution is unique under Assumption 1. Indeed, by supposing that there exist two solutions $\left(\mathbf{u}_{1}, p_{1}\right)$ and $\left(\mathbf{u}_{2}, p_{2}\right)$ of problem $(2.1)$, we obtain that the difference $(\mathbf{w}, \pi):=\left(\mathbf{u}_{2}-\mathbf{u}_{1}, \pi_{2}-\pi_{1}\right)$ solves the following problem

$$
\begin{cases}-\mu \triangle \mathbf{w}+\nabla \pi=\mathbf{0} & \text { a.e. in } \Omega, \\ \nabla \cdot \mathbf{w}=0 & \text { a.e. in } \Omega, \\ \mathbf{w}=\mathbf{0} & \text { on } \Gamma_{D}, \\ \mu \nabla \mathbf{w} \mathbf{n}=\mathbf{0} & \text { on } \Gamma_{N}, \\ \alpha \int_{\Sigma} \mathbf{w} \cdot \mathbf{n} d \sigma+(1-\alpha)\left(c_{2}-c_{1}\right)=0, & \end{cases}
$$

where we have used (2.2) with $c=c_{1}$ and $c=c_{2}$, respectively. Then, by multiplying $(2.5)_{1}$ by $\mathbf{w}$, integrating over $\Omega$, integrating by parts and using Assumption 1 and condition $(2.5)_{5}$, we get

$$
\mu\|\nabla \mathbf{w}\|^{2}=-\left(c_{2}-c_{1}\right)^{2} \frac{1-\alpha}{\alpha},
$$

which leads to $\mathbf{w} \equiv \mathbf{0}$ and $c_{2}=c_{1}$.

Remark 2.2. Even if the splitting given by (2.3) and (2.4) gives us a direct way to solve problem (2.1), this construction may be computational expensive when we apply defective boundary conditions on more than one boundary section. Moreover, it is not trivially extensible to the time-dependent or nonlinear problems. This justifies the search of alternative strategies, which are described in the following sections. 


\section{Variational strategies}

In this Section we review four strategies in view of the numerical solution of system (2.1). To this aim, we set

$$
V:=\left\{v \in H^{1}(\Omega):\left.v\right|_{\Gamma_{D}}=0\right\},
$$

and use the following notation for scalar functions $v$ and $w$, vector functions $\mathbf{g}$ and $\mathbf{h}$, and tensor functions $A$ and $B$

$$
\begin{gathered}
(v, w):=\int_{\Omega} v w d \mathbf{x}, \quad(\mathbf{g}, \mathbf{h}):=\sum_{j} \int_{\Omega} \mathbf{g}_{j} \mathbf{h}_{j} d \mathbf{x}, \quad(A, B):=\sum_{i, j} \int_{\Omega} A_{i j} B_{i j} d \mathbf{x}, \\
\|v\|_{\Sigma}^{2}:=\left(\int_{\Sigma} v d \sigma\right)^{2}, \quad\|\mathbf{g}\|_{\Sigma}^{2}:=\left(\int_{\Sigma} \mathbf{g} \cdot \mathbf{n} d \sigma\right)^{2},
\end{gathered}
$$

and the $L^{2}(\Omega)-$ norms $\|\cdot\|$ follow as usual. We introduce also the following bilinear forms

$$
a(\mathbf{v}, \mathbf{w}):=\mu \int_{\Omega} \nabla \mathbf{v}: \nabla \mathbf{w} d \sigma, \quad b(q, \mathbf{v}):=-\int_{\Omega} q \nabla \cdot \mathbf{v} d \sigma .
$$

In view of the algebraic setting, we introduce two inf-sup compatible finite element spaces $V_{h} \subset V$ and $Q_{h} \subset Q$, whose basis functions are $\varphi_{i}$ and $\psi_{l}$, respectively. We use also the following algebraic variables: $A_{i j}:=$ $a\left(\boldsymbol{\varphi}_{j}, \boldsymbol{\varphi}_{i}\right), B_{i l}:=b\left(\psi_{l}, \boldsymbol{\varphi}_{i}\right),\left(M_{\Sigma}\right)_{i j}:=\int_{\Sigma} \boldsymbol{\varphi}_{j} \cdot \mathbf{n} d \sigma \int_{\Sigma} \boldsymbol{\varphi}_{i} \cdot \mathbf{n} d \sigma, \mathbf{F}_{i}:=\left(\mathbf{f}, \boldsymbol{\varphi}_{i}\right)$ and $\left(\mathbf{F}_{\Sigma}\right)_{i}:=\int_{\Sigma} \boldsymbol{\varphi}_{i} \cdot \mathbf{n} d \sigma$. Finally, with $\mathbf{U}_{i}$ and $\mathbf{P}_{i}$ we indicate the components of the unknown velocity and pressure vectors.

\subsection{Classical variational approach}

This strategy is based on writing the weak formulation of problem (2.1) and by exploiting Assumption 1 (see [20]). We consider first the case $\delta=1$. We observe that for $\alpha \in[0,1)$, thanks to Assumption 1, from condition (1.1) we have

$$
c=\frac{M}{1-\alpha}-\frac{\alpha \rho_{f}}{1-\alpha} \int_{\Sigma} \mathbf{u} \cdot \mathbf{n} d \sigma .
$$

From the momentum equation, we have, for all $\mathbf{v} \in \mathbf{V}$,

$$
(\nabla \mathbf{u}, \nabla \mathbf{v})-(p, \nabla \cdot \mathbf{v})+\int_{\Sigma}(p \mathbf{n}-\mu \nabla \mathbf{u} \mathbf{n}) \cdot \mathbf{v} d \sigma=(\mathbf{f}, \mathbf{v})
$$

where we have exploited the homogeneous boundary conditions on $\partial \Omega \backslash \Sigma$. Then, thanks to Assumption 1, we have

$$
(\nabla \mathbf{u}, \nabla \mathbf{v})-(p, \nabla \cdot \mathbf{v})-c \int_{\Sigma} \mathbf{v} \cdot \mathbf{n} d \sigma=(\mathbf{f}, \mathbf{v}),
$$

which leads, owing to (3.1), to the following variational formulation, holding for $\alpha \in[0,1)$ : 
Given two scalars $\alpha \in[0,1)$ and $M$ and $\mathbf{f} \in \mathbf{L}^{2}(\Omega)$, find $\mathbf{u} \in \mathbf{V}$ and $p \in Q$ such that

$\left\{\begin{array}{l}a(\mathbf{u}, \mathbf{v})+b(p, \mathbf{v})+\frac{\alpha \rho_{f}}{1-\alpha} \int_{\Sigma} \mathbf{u} \cdot \mathbf{n} d \sigma \int_{\Sigma} \mathbf{v} \cdot \mathbf{n} d \sigma=(\mathbf{f}, \mathbf{v})+\frac{M}{1-\alpha} \int_{\Sigma} \mathbf{v} \cdot \mathbf{n} d \sigma \\ b(q, \mathbf{u})=0,\end{array}\right.$

for all $\mathbf{v} \in \mathbf{V}$ and $q \in Q$.

Theorem 3.1. Problem given by (3.2) admits a unique solution.

Proof. By choosing $\|\mathbf{v}\|_{\mathbf{v}}:=\|\nabla \mathbf{v}\|$, the coercivity of the bilinear form at the left hand side of $(3.2)$ is trivially obtained by noticing that $\left(\int_{\Sigma} \mathbf{v} \cdot \mathbf{n} d \sigma\right)^{2} \geq$ 0 , whilst the continuity of this form and of functional at the right hand side follows directly from the trace inequality $\|\mathbf{v}\|_{\Sigma} \lesssim\|\nabla \mathbf{v}\|$ (see, e.g., [14]). The related inf-sup condition follows also immediately by means of classical arguments.

In the case $\alpha=0$, from problem (3.2) we obtain the so-called do-nothing formulation, proposed in [7] for the prescription of a mean pressure condition, given by

$$
\frac{1}{|\Sigma|} \int_{\Sigma}(-p+\delta \mu(\nabla \mathbf{u} \mathbf{n}) \cdot \mathbf{n}) d \sigma=M
$$

with $\delta=0$. However, as pointed out in [18], this formulation is consistent just for the case $\delta=1$. In general, we notice that the case $\delta=0$ can not be treated for any value of $\alpha$, since it would lead to a non consistent formulation. We also observe that with this approach is not possible to treat the case $\alpha=1$ since (3.1) does not hold anymore.

From the algebraic point of view, we obtain the following linear system

$$
\left[\begin{array}{cc}
A+|\Sigma| \frac{\alpha \rho_{f}}{1-\alpha} M_{\Sigma} & B^{T} \\
B & 0
\end{array}\right]\left[\begin{array}{l}
\mathbf{U} \\
\mathbf{P}
\end{array}\right]=\left[\begin{array}{c}
\mathbf{F}+\frac{|\Sigma| M}{1-\alpha} \mathbf{F}_{\Sigma} \\
\mathbf{0}
\end{array}\right]
$$

This system has the classical form of a saddle-point problem and its resolvability is guaranteed by the compatibility of spaces $\mathbf{V}_{h}$ and $Q_{h}$.

\subsection{Augmented approach}

For $\alpha>0$, we consider the following augmented system

Given $\mathbf{f} \in L^{2}(\Omega)$ and two scalars $M$ and $R$, find $\lambda \in \mathbb{R}, \mathbf{u} \in \mathbf{V}$ and $p \in Q$, such that

$$
\left\{\begin{array}{l}
a(\mathbf{u}, \mathbf{v})+b(p, \mathbf{v})+\lambda \int_{\Sigma} \mathbf{v} \cdot \mathbf{n} d \sigma=(\mathbf{f}, \mathbf{v}) \\
b(q, \mathbf{u})=0 \\
\psi \int_{\Sigma} \mathbf{u} \cdot \mathbf{n} d \sigma-\psi \frac{1-\alpha}{\alpha \rho_{f}} \lambda=\psi \frac{M}{\alpha \rho_{f}}
\end{array}\right.
$$

for all $\mathbf{v} \in \mathbf{V}, q \in Q$ and $\psi \in \mathbb{R}$. We observe that we have added one scalar equation and one scalar unknown to the weak formulation of the problem. In the next result, we show the consistency and well-posedness of system (3.3).

Proposition 3.2. System (3.3) admits a unique solution $[\mathbf{u}, p, \lambda]$ for all $\alpha>0$. Moreover, $\mathbf{u}$ and $p$ are also solution of problem (2.1) in a distributional sense. 
Proof. Rewrite system (3.3) as follows

$$
\left\{\begin{aligned}
a(\mathbf{u}, \mathbf{v})+b(p, \mathbf{v})+c(\lambda, \mathbf{v}) & =(\mathbf{f}, \mathbf{v}) \\
b(q, \mathbf{u}) & =0 \\
c(\psi, \mathbf{u})+d(\lambda, \psi) & =\frac{1}{\alpha \rho_{f}} \psi M
\end{aligned}\right.
$$

where $c(\psi, \mathbf{v}):=\psi \int_{\Sigma} \mathbf{v} \cdot \mathbf{n} d \sigma$ and $d(\psi, \zeta):=-\frac{1-\alpha}{\alpha \rho_{f}} \psi \zeta$. It is known that form $b(\cdot, \cdot)$ satisfies an inf-sup condition on $Q \times \mathbf{H}_{0}^{1}(\Omega)$, that is there exists $\beta_{1}>0$ such that for all $q \in Q$, there is a $\mathbf{v}^{*} \in \mathbf{H}_{0}^{1}(\Omega)$ such that

$$
b\left(q, \mathbf{v}^{*}\right) \geq \beta_{1}\left\|\mathbf{v}^{*}\right\|_{H^{1}}\|q\| .
$$

Moreover, in [16] it has been shown that the bilinear form $c(\psi, \mathbf{v})$ satisfies an inf-sup condition when $\mathbf{v}$ is restricted to $\mathbf{V}_{d i v}=\{\mathbf{w} \in \mathbf{V}: b(q, \mathbf{w})=0, \forall \mathbf{w} \in$ $\mathbf{V}\}$, that is there exists $\beta_{2}>0$ such that for all $\psi \in \mathbb{R}$, there is a $\widetilde{\mathbf{v}} \in \mathbf{V}_{\text {div }}$ such that

$$
c(\psi, \widetilde{\mathbf{v}}) \geq \beta_{2}\|\widetilde{\mathbf{v}}\|_{H^{1}}|\psi| .
$$

Now, given any $q \in Q$ and $\psi \in \mathbb{R}$ different from zero, we may take $\mathbf{v}:=\mathbf{v}^{*}+\widetilde{\mathbf{v}}$ to have

$$
b(q, \mathbf{v})+c(\psi, \mathbf{v}) \geq \beta\|\mathbf{v}\|_{V}(\|q\|+|\psi|),
$$

where $\beta:=\min \left\{\beta_{1}, \beta_{2}\right\}$.

Moreover, the bilinear form $d(\cdot, \cdot)$ is always non-positive, for all $\alpha>0$. Therefore, the existence of a unique solution of system (3.3) follows from the general theory of saddle-point problems (see, e.g., [1]).

Take now any $\mathbf{v} \in \mathbf{D}(\Omega)=\mathbf{C}_{0}^{\infty}(\Omega)$. From $(3.3)_{1}$, by contro-integrating by parts, we obtain

$$
<-\mu \triangle \mathbf{u}+\nabla p-\mathbf{f}, \mathbf{v}>=0
$$

by which $-\mu \triangle \mathbf{u}+\nabla p-\mathbf{f}=0$ a.e. in $\Omega$. Analogously, $(3.3)_{2}$ provides $\nabla \cdot \mathbf{u}=0$ a.e. in $\Omega$. If we take now any $\mathbf{v} \in C_{0}^{\infty}(\bar{\Omega})$ with $\left.\mathbf{v}\right|_{\Gamma_{D}}=\mathbf{0}$, and exploit the previous results, we can write (formally) that

$$
\int_{\Sigma \cup \Gamma_{N}}(\mu \nabla \mathbf{u} \cdot \mathbf{n}-p \mathbf{n}) \cdot \mathbf{v} d \gamma+\lambda \int_{\Sigma} \mathbf{v} \cdot \mathbf{n} d \gamma=0,
$$

which, thanks to Assumption 1, implies necessarily that

$$
\lambda=(p \mathbf{n}-\mu \nabla \mathbf{u} \mathbf{n}) \cdot \mathbf{n} \quad \text { on } \Sigma .
$$

Equality (3.4), together with the last of (3.3), tells us that (1.1) is satisfied.

Remark 3.3. In the case $\alpha=1$ we obtain nothing but the Lagrange multipliers approach proposed in [3] to manage a flow rate condition. In this case, the Lagrange multiplier is $\lambda$.

Remark 3.4. By multiplying (3.3) ${ }_{3}$ by $\alpha$ and by taking $\alpha=0$, we obtain from (3.3) two separate blocks, one involving $[\mathbf{u}, p]$ and one $\lambda$. Solving the last equation, we obtain $\lambda=M$, which, substituted in the momentum equation, leads to the do-nothing approach proposed in [7]. 
From the algebraic point view, the augmented formulation with $\delta=1$ and $\alpha>0$ reads

$$
\left[\begin{array}{ccc}
A & B^{T} & \mathbf{F}_{\Sigma}^{T} \\
B & 0 & 0 \\
\mathbf{F}_{\Sigma} & 0 & -\frac{1-\alpha}{\alpha \rho_{f}}
\end{array}\right]\left[\begin{array}{c}
\mathbf{U} \\
\mathbf{P} \\
\Lambda
\end{array}\right]=\left[\begin{array}{c}
\mathbf{F} \\
\mathbf{0} \\
\frac{1}{\alpha \rho_{f}} M
\end{array}\right]
$$

where $\Lambda$ is the approximation of $\lambda$.

In the case $\delta=0$, relation (3.4) still holds, so that system (3.3) for $\alpha>0$ becomes

$$
\left\{\begin{array}{l}
a(\mathbf{u}, \mathbf{v})+b(p, \mathbf{v})+\lambda \int_{\Sigma} \mathbf{v} \cdot \mathbf{n} d \sigma=(\mathbf{f}, \mathbf{v}) \\
b(q, \mathbf{u})=0 \\
\psi \int_{\Sigma} \mathbf{u} \cdot \mathbf{n} d \sigma-\psi \frac{1-\alpha}{|\Sigma| \alpha \rho_{f}} \int_{\Sigma} \mu(\nabla \mathbf{u} \mathbf{n}) \cdot \mathbf{n} d \sigma-\psi \frac{1-\alpha}{\alpha \rho_{f}} \lambda=\psi \frac{M}{\alpha \rho_{f}}
\end{array}\right.
$$

for all $\mathbf{v} \in \mathbf{V}, q \in Q$ and $\psi \in \mathbb{R}$, and the system looses its saddle point structure. In this case the algebraic formulation becomes

$$
\left[\begin{array}{ccc}
A & B^{T} & \mathbf{F}_{\Sigma}^{T} \\
B & 0 & 0 \\
\mathbf{F}_{\Sigma}-\frac{1-\alpha}{\alpha \rho_{f}} G & 0 & -\frac{1-\alpha}{\alpha \rho_{f}}
\end{array}\right]\left[\begin{array}{c}
\mathbf{U} \\
\mathbf{P} \\
\Lambda
\end{array}\right]=\left[\begin{array}{c}
\mathbf{F} \\
\mathbf{0} \\
\frac{M}{\alpha \rho_{f}}
\end{array}\right]
$$

with $G_{i}:=\frac{1}{|\Sigma|} \int_{\Sigma} \mu\left(\nabla \varphi_{i} \mathbf{n}\right) \cdot \mathbf{n} d \sigma$.

\subsection{Control-based approach}

In this Section we propose to prescribe condition (1.1) through the minimization of a suitable functional and the choice of a proper control variable, as done in the optimal control approach for PDE's. In particular, we generalize here what done in [4] for the particular cases $\alpha=0$ and $\alpha=1$.

3.3.1. Minimization problem. The idea is based on Assumption 1, that is on the existence on a unique (but unknown) scalar $c$ which guarantees the satisfaction of (1.1). Therefore, we can think to look for the constant Neumann boundary condition $k \in \mathcal{A}$, which plays the role of control variable, such that the distance between the left hand side of (1.1) and the datum $M$ is minimal in some norm. Let $\mathcal{A}$ be the admissible set for the control variable, given by $\mathcal{A}:=\{k \in \mathbb{R}:|k| \leq C\}$, for a given constant $C>0$. We introduce the following functional

$\mathcal{J}(\mathbf{v}, q ; k):=\frac{1}{2}\left(\rho_{f} \alpha \int_{\Sigma} \mathbf{v} \cdot \mathbf{n} d \sigma+\frac{1-\alpha}{|\Sigma|} \int_{\Sigma}(-p+\delta \mu(\nabla \mathbf{u} \mathbf{n}) \cdot \mathbf{n}) d \sigma-M\right)^{2}$,

and we consider the following minimization problem.

Problem 1. Given two scalars $\alpha \in[0,1]$ and $M$ and $\mathbf{f} \in \mathbf{L}^{2}(\Omega)$, find $\mathbf{u} \in$ $\mathbf{V}, p \in Q$ and $k \in \mathcal{A}$, which satisfy

$$
\min _{\mathbf{v}, q \in \mathbf{V} \times Q} \mathcal{J}(\mathbf{v}, q ; k),
$$

under the constraint given by the state problem

$$
\left\{\begin{array}{l}
a(\mathbf{u}, \mathbf{v})+b(p, \mathbf{v})+k \int_{\Sigma} \mathbf{v} \cdot \mathbf{n} d \sigma=(\mathbf{f}, \mathbf{v}) \\
b(q, \mathbf{u})=0
\end{array}\right.
$$


for all $\mathbf{v} \in \mathbf{V}$ and $q \in Q$.

We observe that functional $\mathcal{J}$ depends, through the solution of the state problem, on the control variable $k$.

We have the following

Theorem 3.5. Under the assumption that $\mathbf{u} \in \mathbf{H}^{2}(\Omega) \cap \mathbf{V}$ and $p \in H^{1}(\Omega)$, there exists at least a minimizer to the optimization Problem 1.

Proof. For a given $k \in \mathcal{A}$, from $\mathbf{f} \in \mathbf{L}^{2}(\Omega)$, the assumption $\mathbf{u} \in \mathbf{H}^{2}(\Omega) \cap \mathbf{V}$ and $p \in H^{1}(\Omega)$ is satisfied under some restrictions on the regularity of $\partial \Omega$, see, e.g., [6]. In particular, the solution of the state problem depends with continuity on the data (and in particular on $k$ ). Indeed, we have

$$
\|\mathbf{u}\|_{\mathbf{H}^{2}(\Omega)}+\|p\|_{H^{1}(\Omega)} \lesssim\|\mathbf{f}\|_{\mathbf{L}^{2}(\Omega)}+|k| \leq\|\mathbf{f}\|_{\mathbf{L}^{2}(\Omega)}+C .
$$

Then, we can build two maps $\mathbf{u}: \mathbb{R} \rightarrow \mathbf{H}^{2}(\Omega) \cap \mathbf{V}$ and $p: \mathbb{R} \rightarrow H^{1}(\Omega)$ which, given a scalar $k$, return $\mathbf{u}=\mathbf{u}(k)$ and $p=p(k)$ solutions of the state problem. Thus we can set $\mathcal{J}(k)=\mathcal{J}(\mathbf{u}(k), p(k) ; k)$.

For $k \in \mathcal{A}$, we observe that the fact that $\mathbf{u} \in \mathbf{H}^{2}(\Omega)$ and $p \in H^{1}(\Omega)$ implies, in particular, $\left.\mathbf{u} \cdot \mathbf{n}\right|_{\Sigma} \in L^{2}(\Sigma),\left.(\nabla \mathbf{u} \mathbf{n}) \cdot \mathbf{n}\right|_{\Sigma} \in L^{2}(\Sigma)$ and $\left.p\right|_{\Sigma} \in L^{2}(\Sigma)$. Then $\mathcal{J}$ is bounded and $\inf _{k \in \mathcal{A}} \mathcal{J}(k) \in \mathbb{R}$, so that there exists a sequence $k^{n} \in \mathcal{A}$ (converging to $\bar{k} \in \mathcal{A}$ ) such that $\lim _{n \rightarrow \infty} \mathcal{J}\left(k^{n}\right)=\inf _{k \in \mathcal{A}} \mathcal{J}(k)$. Since the bound (3.6) on $\mathbf{u}^{n}:=\mathbf{u}\left(k^{n}\right)$ and $p^{n}:=p\left(k^{n}\right)$ is independent of $k^{n}$, there exists two sub-sequences (denoted again with $\mathbf{u}^{n}$ and $p^{n}$ ) weakly converging to $\overline{\mathbf{u}} \in \mathbf{H}^{2}(\Omega)$ in the $H^{2}$ norm and to $\bar{p} \in H^{1}(\Omega)$ in the $H^{1}$ norm, respectively. It is easy to see that $\overline{\mathbf{u}}$ and $\bar{p}$ satisfy the state problem with $\bar{k}$ as Neumann condition on $\Sigma$.

Now, the weak convergence of $\mathbf{u}^{n}$ in $\mathbf{H}^{2}(\Omega)$ implies the strong convergence in $\mathbf{H}^{1}(\Sigma)$, which in particular implies that both $\mathbf{u}^{n} \cdot \mathbf{n}$ and $\left(\nabla \mathbf{u}^{n} \mathbf{n}\right) \cdot \mathbf{n}$ strongly converge in $L^{2}(\Sigma)$. In the same way, we observe that the weak convergence of $p^{n}$ in $H^{1}(\Omega)$ implies the strong convergence in $L^{2}(\Sigma)$. Then, we have

$$
\begin{aligned}
& \mathcal{J}(\bar{k})=\frac{1}{2}\left(\rho_{f} R \int_{\Sigma} \overline{\mathbf{u}} \cdot \mathbf{n} d \sigma+\frac{1}{|\Sigma|} \int_{\Sigma}(-\bar{p}+\delta \mu(\nabla \overline{\mathbf{u}} \mathbf{n}) \cdot \mathbf{n}) d \sigma-M\right)^{2}= \\
& =\frac{1}{2} \lim _{n \rightarrow \infty}\left(\rho_{f} R \int_{\Sigma} \mathbf{u}\left(k^{n}\right) \cdot \mathbf{n} d \sigma+\frac{1}{|\Sigma|} \int_{\Sigma}\left(-p\left(k^{n}\right)+\delta \mu\left(\nabla \mathbf{u}\left(k^{n}\right) \mathbf{n}\right) \cdot \mathbf{n}\right) d \sigma-M\right)^{2} \leq \\
& =\lim _{n \rightarrow \infty} \mathcal{J}\left(k^{n}\right)=\inf _{k \in \mathcal{A}} \mathcal{J}(k),
\end{aligned}
$$

which shows that $\bar{k}$ realizes the infimum of $\mathcal{J}$. This concludes the proof.

Remark 3.6. In [4], the authors provided, through a different proof, an existence result just for the case $\alpha=0$. Here we have provided a new result which holds for all values of $\alpha$.

3.3.2. First order optimality conditions. In view of the numerical solution of Problem 1, we follow the standard Lagrange multiplier approach. By considering the Lagrangian functional, obtained by adding to $\mathcal{J}$ the state problem (3.5) as a constraint, and by imposing that its gradient is zero, we obtain the Karush-Kuhn-Tucker (KKT) conditions, formed by the state and the adjoint 
problems and by the optimality condition, as follows:

Given $\mathbf{f} \in \mathbf{L}^{2}(\Omega)$ and two scalars $M$ and $\alpha$, find $k \in \mathcal{A}$, $\mathbf{u} \in \mathbf{V}, p \in$ $L^{2}(\Omega), \lambda_{u} \in \mathbf{V}$ and $\lambda_{p} \in L^{2}(\Omega)$, such that

$$
\begin{aligned}
& \text { State pbl : }\left\{\begin{array}{l}
a(\mathbf{u}, \mathbf{v})+b(p, \mathbf{v})+k \int_{\Sigma} \mathbf{v} \cdot \mathbf{n} d \sigma=(\mathbf{f}, \mathbf{v}), \\
b(q, \mathbf{u})=0 ;
\end{array}\right. \\
& \text { Adj pbl : }\left\{\begin{array}{c}
a\left(\mathbf{v}, \boldsymbol{\lambda}_{u}\right)+b\left(\lambda_{p}, \mathbf{v}\right)+ \\
\quad+\left(\rho_{f} \alpha \int_{\Sigma} \mathbf{u} \cdot \mathbf{n} d \sigma+\frac{1-\alpha}{|\Sigma|} \int_{\Sigma} \delta \mu(\nabla \mathbf{u} \cdot \mathbf{n}) \cdot \mathbf{n} d \sigma\right) \times \\
\times\left(\rho_{f} \alpha \int_{\Sigma} \mathbf{v} \cdot \mathbf{n} d \sigma+\frac{1-\alpha}{|\Sigma|} \int_{\Sigma} \delta \mu(\nabla \mathbf{v} \cdot \mathbf{n}) \cdot \mathbf{n} d \sigma\right)+ \\
-\frac{1-\alpha}{|\Sigma|} \int_{\Sigma} p d \sigma\left(\rho_{f} \alpha \int_{\Sigma} \mathbf{v} \cdot \mathbf{n} d \sigma+\frac{1-\alpha}{|\Sigma|} \int_{\Sigma} \delta \mu(\nabla \mathbf{v} \cdot \mathbf{n}) \cdot \mathbf{n} d \sigma\right)= \\
=M\left(\rho_{f} \alpha \int_{\Sigma} \mathbf{v} \cdot \mathbf{n} d \sigma+\frac{1-\alpha}{|\Sigma|} \int_{\Sigma} \delta \mu(\nabla \mathbf{v} \cdot \mathbf{n}) \cdot \mathbf{n} d \sigma\right), \\
b\left(q, \lambda_{u}\right)+ \\
-\left(\rho_{f} \alpha \int_{\Sigma} \mathbf{u} \cdot \mathbf{n} d \sigma+\frac{1-\alpha}{|\Sigma|} \int_{\Sigma} \delta \mu(\nabla \mathbf{u} \cdot \mathbf{n}) \cdot \mathbf{n} d \sigma\right) \frac{1-\alpha}{|\Sigma|} \int_{\Sigma} q d \sigma+ \\
+\frac{1-\alpha}{|\Sigma|} \int_{\Sigma} p d \sigma \frac{1-\alpha}{|\Sigma|} \int_{\Sigma} q d \sigma=-M \frac{1-\alpha}{|\Sigma|} \int_{\Sigma} q d \sigma ;
\end{array}\right.
\end{aligned}
$$

Opt. cond : $\int_{\Sigma} \boldsymbol{\lambda}_{u} \cdot \mathbf{n} d \sigma=0$,

for all $\mathbf{v} \in \mathbf{V}$ and $q \in Q$, and where $\boldsymbol{\lambda}_{u}$ and $\lambda_{p}$ are the Lagrange multipliers. We remark that this formulation holds for all values of $\alpha \in[0,1]$ and $\delta$.

From the algebraic point of view, system (3.7) becomes

$$
\left[\begin{array}{ccccc}
A & B^{T} & 0 & 0 & \mathbf{F}_{\Sigma}^{T} \\
B & 0 & 0 & 0 & 0 \\
C & D & A & B^{T} & 0 \\
D^{T} & \frac{(1-\alpha)^{2}}{|\Sigma|^{2}} M_{\Sigma}^{P} & B & 0 & 0 \\
0 & 0 & \mathbf{F}_{\Sigma} & 0 & 0
\end{array}\right]\left[\begin{array}{c}
\mathbf{U} \\
\mathbf{P} \\
\boldsymbol{\Lambda}_{u} \\
\boldsymbol{\Lambda}_{p} \\
K
\end{array}\right]=\left[\begin{array}{c}
\mathbf{F} \\
\mathbf{0} \\
M\left(\rho_{f} \alpha \mathbf{F}_{\Sigma}-\frac{1-\alpha}{|\Sigma|} \mathbf{H}_{\Sigma}\right) \\
M \frac{1-\alpha}{|\Sigma|} \mathbf{F}_{\Sigma}^{P} \\
0
\end{array}\right]
$$

where $C=\rho_{f}^{2} \alpha^{2} M_{\Sigma}+\delta \frac{\rho_{f} \alpha(1-\alpha)}{|\Sigma|}\left(T_{\Sigma}+T_{\Sigma}^{T}\right)+\delta^{2} \frac{(1-\alpha)^{2}}{|\Sigma|^{2}} K_{\Sigma}$,

$D=-\frac{\rho_{f} \alpha(1-\alpha)}{|\Sigma|} N_{\Sigma}-\delta \frac{(1-\alpha)^{2}}{|\Sigma|^{2}} S_{\Sigma},\left(T_{\Sigma}\right)_{i j}:=\int_{\Sigma} \boldsymbol{\varphi}_{j} \cdot \mathbf{n} d \sigma \int_{\Sigma} \mu\left(\nabla \boldsymbol{\varphi}_{i} \mathbf{n}\right) \cdot \mathbf{n} d \sigma$, $\left(K_{\Sigma}\right)_{i j}:=\int_{\Sigma} \mu\left(\nabla \boldsymbol{\varphi}_{j} \mathbf{n}\right) \cdot \mathbf{n} d \sigma \int_{\Sigma} \mu\left(\nabla \boldsymbol{\varphi}_{i} \mathbf{n}\right) \cdot \mathbf{n} d \sigma$ $\left(N_{\Sigma}\right)_{i l}:=\int_{\Sigma} \psi_{l} d \sigma \int_{\Sigma} \boldsymbol{\varphi}_{i} \cdot \mathbf{n} d \sigma,\left(S_{\Sigma}\right)_{i l}:=\int_{\Sigma} \psi_{l} d \sigma \int_{\Sigma} \mu\left(\nabla \boldsymbol{\varphi}_{i} \mathbf{n}\right) \cdot \mathbf{n} d \sigma$, $\left(M_{\Sigma}^{P}\right)_{l m}:=\int_{\Sigma} \psi_{m} d \sigma \int_{\Sigma} \psi_{l} d \sigma,\left(\mathbf{H}_{\Sigma}\right)_{i}:=\int_{\Sigma} \mu\left(\nabla \boldsymbol{\varphi}_{i} \mathbf{n}\right) \cdot \mathbf{n} d \sigma,\left(\mathbf{F}_{\Sigma}^{P}\right)_{l}:=\int_{\Sigma} \psi_{l} d \sigma$, and where $\left(\boldsymbol{\Lambda}_{u}\right)_{i}$ and $\left(\boldsymbol{\Lambda}_{p}\right)_{i}$ are the components of the finite element approximations of the Lagrange multipliers, and $K$ the approximation of the control variable $k$.

\subsection{Approach based on the Nitsche method}

The last strategy we consider has been originally proposed in [18], and it is based on the Nitsche method [9] for general boundary conditions (see $[8,11])$.

Let $h<1$ be a parameter, which in the finite element setting will be a characteristic mesh size. We first consider the case $\delta=1$. Then, for a given 
penalization parameter $\gamma>0$, we introduce the following bilinear forms:

$$
\begin{aligned}
& a^{N}(\mathbf{u}, \mathbf{v})=a(\mathbf{u}, \mathbf{v})+\frac{\rho_{f} \alpha}{1-\alpha\left(1-\rho_{f} \gamma h\right)} \frac{1}{|\Sigma|} \int_{\Sigma} \mathbf{u} \cdot \mathbf{n} d \sigma \int_{\Sigma} \mathbf{v} \cdot \mathbf{n} d \sigma+ \\
& -\frac{\rho_{f} \alpha \gamma h}{1-\alpha\left(1-\rho_{f} \gamma h\right)} \frac{1}{|\Sigma|}\left[\int_{\Sigma} \mu(\nabla \mathbf{u} \mathbf{n}) \cdot \mathbf{n} d \sigma \int_{\Sigma} \mathbf{v} \cdot \mathbf{n} d \sigma+\int_{\Sigma} \mu(\nabla \mathbf{v} \mathbf{n}) \cdot \mathbf{n} d \sigma \int_{\Sigma} \mathbf{u} \cdot \mathbf{n} d \sigma\right]+ \\
& \quad-\frac{(1-\alpha) \gamma h}{1-\alpha\left(1-\rho_{f} \gamma h\right)} \frac{1}{|\Sigma|} \int_{\Sigma} \mu(\nabla \mathbf{u} \mathbf{n}) \cdot \mathbf{n} d \sigma \int_{\Sigma} \mu(\nabla \mathbf{v} \mathbf{n}) \cdot \mathbf{n} d \sigma \\
& b^{N}(q, \mathbf{v})=b(q, \mathbf{v})+\frac{\rho_{f} \alpha \gamma h}{1-\alpha\left(1-\rho_{f} \gamma h\right)} \frac{1}{|\Sigma|} \int_{\Sigma} q d \sigma \int_{\Sigma} \mathbf{v} \cdot \mathbf{n} d \sigma+ \\
& +\frac{(1-\alpha) \gamma h}{1-\alpha\left(1-\rho_{f} \gamma h\right)} \frac{1}{|\Sigma|} \int_{\Sigma} q d \sigma \int_{\Sigma} \mu(\nabla \mathbf{v} \mathbf{n}) \cdot \mathbf{n} d \sigma \\
& c^{N}(p, q)=-\frac{(1-\alpha) \gamma h}{1-\alpha\left(1-\rho_{f} \gamma h\right)} \frac{1}{|\Sigma|} \int_{\Sigma} p d \sigma \int_{\Sigma} q d \sigma
\end{aligned}
$$

and the following linear functionals:

$$
\begin{aligned}
F^{N}(\mathbf{v}) & =\int_{\Omega} \mathbf{f} \cdot \mathbf{v} d \mathbf{x}+\frac{1-\alpha}{1-\alpha\left(1-\rho_{f} \gamma h\right)} \frac{1}{|\Sigma|} M \int_{\Sigma} \mathbf{v} \cdot \mathbf{n} d \sigma+ \\
& -\frac{(1-\alpha) \gamma h}{1-\alpha\left(1-\rho_{f} \gamma h\right)} \frac{1}{|\Sigma|} M \int_{\Sigma} \mu(\nabla \mathbf{v} \mathbf{n}) \cdot \mathbf{n} d \sigma+ \\
G^{N}(q) & =\frac{(1-\alpha) \gamma h}{1-\alpha\left(1-\rho_{f} \gamma h\right)} \frac{1}{|\Sigma|} M \int_{\Sigma} q d \sigma .
\end{aligned}
$$

We consider the following discrete problem:

Problem 2. Given $\mathbf{f} \in \mathbf{L}^{2}(\Omega)$ and $M \in \mathbb{R}$, find $\mathbf{u}_{h} \in \mathbf{V}_{h}$ and $p_{h} \in Q_{h}$ such that

$$
\left\{\begin{array}{l}
a^{N}\left(\mathbf{u}_{h}, \mathbf{v}_{h}\right)+b^{N}\left(p_{h}, \mathbf{v}_{h}\right)=F\left(\mathbf{v}_{h}\right) \quad \forall \mathbf{v}_{h} \in \mathbf{V}_{h}, \\
b^{N}\left(q_{h}, \mathbf{u}_{h}\right)+c^{N}\left(p_{h}, q_{h}\right)=G(q) \quad \forall q_{h} \in Q_{h} .
\end{array}\right.
$$

We introduce the following norm

$$
\|\mathbf{v}\|_{h}^{2}:=\|\nabla \mathbf{v}\|^{2}+\frac{\rho_{f} \alpha}{1-\alpha\left(1-\rho_{f} \gamma h\right)}|\mathbf{v} \cdot \mathbf{n}|_{\Sigma}^{2}, \quad \mathbf{v} \in \mathbf{H}^{1}(\Omega) .
$$

All the following analysis refers to norm (3.12) and to constants independent of $h$ and $R$.

Theorem 3.7. Suppose that an inf-sup condition holds for the classical Stokes problem with the velocity field restricted to $\mathbf{V}_{h} \cap \mathbf{H}_{0}^{1}(\Omega)$, that is there exists $\beta>0$ such that for all $q \in Q_{h}$, there is a $\mathbf{v} \in \mathbf{V}_{h} \cap \mathbf{H}_{0}^{1}(\Omega)$ such that $\int_{\Sigma} q \nabla \cdot \mathbf{v} d \sigma \geq \beta\|q\|\|\nabla \mathbf{v}\|$ (this assumption holds true, for example for the Taylor-Hood and the MINI elements, see [2]). Then, formulation (3.11) is consistent with problem (2.1), and admits a unique solution. Moreover, the convergence of the numerical solution towards the continuous one is optimal. 
A proof may be found in [18].

The corresponding algebraic formulation reads

$$
\left[\begin{array}{cc}
A^{N} & \left(B^{N}\right)^{T} \\
B^{N} & C^{N}
\end{array}\right]\left[\begin{array}{l}
\mathbf{U} \\
\mathbf{P}
\end{array}\right]=\left[\begin{array}{c}
\mathbf{F}^{N} \\
\mathbf{F}_{\Sigma}^{P}
\end{array}\right],
$$

where $A_{i j}^{N}:=A_{i j}+\frac{\rho_{f} \alpha}{1-\alpha\left(1-\rho_{f} \gamma h\right)} \frac{1}{|\Sigma|} M_{\Sigma, i j}-\frac{\rho_{f} \alpha \gamma h}{1-\alpha\left(1-\rho_{f} \gamma h\right)} \frac{1}{|\Sigma|}\left(T_{\Sigma, i j}+T_{\Sigma, j i}\right)-$ $\frac{(1-\alpha) \gamma h}{1-\alpha\left(1-\rho_{f} \gamma h\right)} \frac{1}{|\Sigma|} K_{\Sigma, i j}$,

$B_{i l}^{N}:=B_{i l}+\frac{\rho_{f} \alpha \gamma h}{1-\alpha\left(1-\rho_{f} \gamma h\right)} \frac{1}{|\Sigma|} N_{\Sigma, i l}+\frac{(1-\alpha) \gamma h}{1-\alpha\left(1-\rho_{f} \gamma h\right)} \frac{1}{|\Sigma|} S_{\Sigma, i l}, C_{l m}^{N}:=-\frac{(1-\alpha) \gamma h}{1-\alpha\left(1-\rho_{f} \gamma h\right)} \frac{1}{|\Sigma|} M_{\Sigma, l m}^{P}$, and

$\mathbf{F}_{i}^{N}:=\mathbf{F}_{i}+\frac{1-\alpha}{1-\alpha\left(1-\rho_{f} \gamma h\right)} \frac{1}{|\Sigma|} M \mathbf{F}_{\Sigma, i}-\frac{(1-\alpha) \gamma h}{1-\alpha\left(1-\rho_{f} \gamma h\right)} \frac{1}{|\Sigma|} M \mathbf{H}_{\Sigma, i}$.

In the case of $\delta=\alpha=0$, in [18] it has been proposed to substitute form $a^{N}(\cdot, \cdot)$ and functional $F(\cdot)$ with

$$
\begin{aligned}
& a^{P}(\mathbf{u}, \mathbf{v}):=a(\mathbf{u}, \mathbf{v})-\frac{1}{|\Sigma|} \int_{\Sigma} \mu(\nabla \mathbf{u} \mathbf{n}) \cdot \mathbf{n} d \sigma \int_{\Sigma} \mathbf{v} \cdot \mathbf{n} d \sigma, \\
& F^{P}(\mathbf{v})=\int_{\Sigma} \mathbf{f} \cdot \mathbf{v} d \sigma-P \int_{\Sigma} \mathbf{v} \cdot \mathbf{n} d \sigma .
\end{aligned}
$$

We observe that this formulation is consistent with problem (2.1) with $\delta=0$ and $\alpha=0$.

\section{Conclusions}

In this work we have presented different strategies to prescribe a general defective boundary condition to the Stokes problem. In particular, we have extended to a more general setting $(\alpha \in(0,1))$ the Lagrange multipliers approach presented in [3] and the control-based approach introduced in [4].

All of such strategies have their advantages and disadvantages. First of all, we have noticed that some of them cannot be applied in the whole range of values of $\alpha$ and $\delta$. In Table 1 we summarize the applicability of the four different methodologies described in this work to the different cases.

\begin{tabular}{c||c|c|c|c|c} 
& $\alpha=0$ & $\alpha=0$ & $0<\alpha<1$ & $0<\alpha<1$ & $\alpha=1$ \\
& $\delta=0$ & $\delta=1$ & $\delta=0$ & $\delta=1$ & \\
\hline Classical & & OK & & OK & \\
Augmented & & OK & OK & OK & OK \\
Control & OK & OK & OK & OK & OK \\
Nitsche & OK & OK & & OK & OK
\end{tabular}

TABLE 1. Applicability of the different methods leading to a consistent formulation.

We can notice that the control-based approach is most general but generally is the most expensive one computationally. The Nitsche approach is rather general and does not introduce any additional unknown, but the 
boundary condition is satisfied only approximately and needs the tuning of the penalization parameter.

From the applicative point of view, it is also important to consider which methods could be implemented using existing solvers without modifications (modularity). We have not dealt this specific aspect in this work. Yet, from our experience the Augmented and the Control approaches are the ones that lend themselves better to a modular implementation than the others $[16,4]$.

\section{References}

[1] M. Benzi, H. G.H. Golub, and J. Liesen. Numerical solution of saddle point problems. Acta Numerica, pages 1-137, 2005.

[2] F. Brezzi and M. Fortin. Mixed and hybrid finite element methods. Springer Verlag, 1991.

[3] L. Formaggia, J.-F. Gerbeau, F. Nobile, and A. Quarteroni. Numerical treatment of defective boundary conditions for the Navier-Stokes equation. SIAM Journal on Numerical Analysis, 40(1):376-401, 2002.

[4] L. Formaggia, A. Veneziani, and C. Vergara. A new approach to numerical solution of defective boundary value problems in incompressible fluid dynamics. SIAM Journal on Numerical Analysis, 46(6):2769-2794, 2008.

[5] L. Formaggia, A. Veneziani, and C. Vergara. Flow rate boundary problems for an incompressible fluid in deformable domains: formulations and solution methods. Computer Methods in Applied Mechanics and Engineering, 199 (912):677-688, 2010.

[6] V. Girault and P.A. Raviart. Finite element methods for Navier-Stokes equations. Springer-Verlag, 1986.

[7] J.G. Heywood, R. Rannacher, and S. Turek. Artificial boundaries and flux and pressure conditions for the incompressible Navier-Stokes equations. International Journal for Numerical Methods in Fluids, 22:325-352, 1996.

[8] M. Juntunen and R. Stenberg. Nitsche's method for general boundary conditions. Math. Comp., 78:1353-1374, 2009.

[9] J.A. Nitsche. Uber ein variationsprinzip zur lozung von dirichlet-problemen bei verwendung von teilraumen, die keinen randbedingungen unterworfen sind. Abhandlungen aus dem Mathematischen Seminar der Universitat Hamburg, 36:9-15, 1970/71.

[10] K. Perktold, R.M. Nerem, and R.O. Peter. A numerical calculation of flow in a curved tube model of the left main coronary artery. Journal of Biomechanics, 24:175-189, 1991.

[11] A. Porpora, P. Zunino, C. Vergara, and M. Piccinelli. Numerical treatment of boundary conditions to replace lateral branches in haemodynamics. Int J Num Meth Biomed Eng, accepted for pubblication.

[12] A. Quarteroni, M. Tuveri, and A. Veneziani. Computational vascular fluid dynamics: Problems, models and methods. Computing and Visualisation in Science, 2:163-197, 2000.

[13] D. Steinman and C. Taylor. Flow imaging and computing: Large artery hemodynamics. Annals Biomed. Eng., 33(12):1704-1709, 2005. 
[14] V. Thomée. Galerkin finite element method for parabolic problems. Springer, Springer series in computational matehmatics, vol. 25, 2001.

[15] A. Veneziani. Mathematical and Numerical Modeling of Blood Flow Problems. PhD thesis, University of Milan, 1998.

[16] A. Veneziani and C. Vergara. Flow rate defective boundary conditions in haemodinamics simulations. International Journal for Numerical Methods in Fluids, 47:803-816, 2005.

[17] C. Vergara. Numerical Modeling of Defective Boundary Problems in Incompressible Fluid dynamics. Applications to Computational Haemodynamics. $\mathrm{PhD}$ thesis, MOX - Politecnico di Milano, 2006.

[18] C. Vergara. Nitsche's method for defective boundary value problems in incompressible fluid-dynamics. Journal of Scientific Computing, 46:100-123, 2011.

[19] C. Vergara, F. Viscardi, L. Antiga, and G.B. Luciani. Influence of bicuspid valve geometry on ascending aortic fluid-dynamics: a parametric study. Artificial Organs, 2011.

[20] I. Vignon-Clementel, C. Figueroa, K. Jansen, and C. Taylor. Outflow boundar conditions for the three-dimensional finite element modeling of blood flow and pressure in arteries. Computer Methods in Applied Mechanics and Engineering, 195:3776-3796, 2006.

[21] P. Zunino. Numerical approximation of incompressible flows with net flux defective boundary conditions by means of penalty technique. Computer Methods in Applied Mechanics and Engineering, 198(37-40):3026-3038, 2009.

Luca Formaggia

MOX, Dipartimento di Matematica, Politecnico di Milano

Piazza Leonardo da Vinci 32

20133 Milano

Italy

e-mail: luca.formaggia@polimi.it

Christian Vergara

Dipartimento di Ingegneria dell'Informazione e Metodi Matematici, Università di Bergamo

Viale Marconi 5

24044 Dalmine (BG)

Italy

e-mail: christian.vergara@unibg.it 